\title{
SCHWEBENDE INFRASTRUKTUREN
}

\author{
Die extraterritorialen Ballons von Project Loon \\ und die Medien der Stratosphäre
}

Laut der International Telecommunication Union hatten 2019 etwa 46 Prozent der Weltbevölkerung keinen Zugang zum Internet. ${ }^{1}$ Um den sogenannten digital divide zu schließen, hat unter Stichworten wie «internet for all» und «connecting the unconnected» in den letzten Jahren ein Wettrennen begonnen, Alternativen zu einem zeit-, energie- und kostenintensiven Ausbau von Glasfasernetzen zu entwickeln. ${ }^{2}$ Die beteiligten Tech-Firmen setzen vor allem auf Internetverbindungen aus dem Welt- oder Luftraum. Einige Unternehmen wie $\mathrm{O}_{3} \mathrm{~b}$, OneWeb und SpaceX greifen die vor knapp 30 Jahren durchgeführten Versuche auf, Internet durch orbitale Satelliten zur Verfügung zu stellen. ${ }^{3}$ Andere Firmen wie Facebook oder Google investieren seit den I99oer Jahren in zunehmend erprobte atmosphärische Satelliten, auch bekannt als PseudoSatelliten oder high-altitude platforms (HAPs), die in etwa 20 Kilometern Höhe jenseits von Flugverkehr und Wettergeschehen in der Stratosphäre fliegen. ${ }^{4}$ Dahinter verbergen sich Ballons, Luftschiffe und Flugzeuge, die zu Telekommunikations- oder Fernerkundungszwecken genutzt werden.

Der vorliegende Aufsatz beleuchtet eine solche atmosphärische Plattform aus medienwissenschaftlicher Perspektive. Im Zentrum stehen die Internetballons, die Google seit 2012 unter dem Namen «Project Loon» entwickelt. Die Netzwerke dieser Ballons bzw. Loons lassen sich mit Nicole Starosielski als media infrastructures beschreiben, als gleichermaßen materielles und diskursives Informationssystem, dessen technische und soziale Seiten nicht losgelöst voneinander zu betrachten sind. ${ }^{5}$ In den Fokus rückt aus diesem Blickwinkel, dass Googles Mutterkonzern Alphabet Inc. mit dem Projekt gleichzeitig als Telekommunikationsnetzbetreiber und Internetdienstleister agiert. Über unternehmenseigene Netzwerke können jene Leistungen angeboten werden, an denen der Konzern verdient. Mit den Loons, so lässt sich mit Keller Easterling pointieren, wird die extraterritoriale Stratosphäre von einem Privatkonzern als infrastructure space besetzt. Vergleichbar zu anderen großskaligen räumlichen Organisationen wie der Eisenbahn oder Unterseekabeln bilden die Ballons ein
1 Vgl. International Telecommunication Union: Measuring Digital Development. Facts and Figures 2019, Genf 2019, 1.

2 Vgl. Lisa Parks, Matthew Graydon: «Connecting the Unconnected. A Critical Assessment of US Satellite Internet Services, in: Media, Culture and Society, 2019, 1-17, hier 2. 3 Vgl. ebd., 7.

4 Vgl. Flavio Araripe d'Oliveira u. a.: High-Altitude Platforms. Present Situation and Technology Trends, in: Journal of Aerospace Technology and Management, 2016, Bd. 8, Nr. 3, 249-262.

5 Nicole Starosielski: Fixed Flow. Undersea Cables as Media Infrastructure, in: Lisa Parks, Nicole Starosielski (Hg.): Signal Traffic. Critical Studies of Media Infrastructures, Urbana u. a. 2015, 53-70, hier 55. 
6 Vgl. Keller Easterling: Extrastatecraft. The Power of Infrastructure Space, London, Brooklyn 2014, 15.

7 Vgl. Susan Leigh Star, Karen Ruhleder: Steps Toward an Ecology of Infrastructure. Design and Access for Large Information Spaces, in: Information Systems Research, Bd. 7, Nr. 1, 1996, 111-134, hier $111 \mathrm{f}$.

8 Vgl. Michael Kyle Caswell:

The Need for Vertical Delineation of Air and Space. Can Google's Project Loon Survive Without It?, in: Tulane Journal of International and Comparative Law, Bd. 24, Nr. 1 , 2015, 205-235, hier 207.

9 Vgl. ebd.

10 vgl. ebd. dynamisches Raumsystem, in dem sich jenseits bekannter legislativer Prozesse ein verändertes Verhältnis von Wirtschaft und Staat manifestiert. ${ }^{6}$ Googles Ballon-Netzwerke als Medieninfrastruktur zu untersuchen, bedeutet nicht, sie als räumlich und zeitlich klar umgrenzt zu definieren. Vielmehr lassen sie sich als dezentralisierte Technologien betrachten, die über weite geografische Distanzen verwendet werden, globale Standards mit lokalen Besonderheiten verbinden sowie gewachsene Technologien und Praktiken formen und von diesen geformt werden. ${ }^{7}$ Eine rechts-, technik- und wissenshistorisch informierte Perspektive erlaubt es nachzuzeichnen, welche politischen, technischen und epistemischen Konzepte der Stratosphäre Google mit seinen Ballon-Netzwerken aufgreift, verändert oder konstituiert.

\section{Politiken des «Loon for All»}

Seit 20I3 hat Project Loon Hunderte von Ballons in die Stratosphäre geschickt, wo sie weit über Flugzeugen, der Tierwelt und dem Wettergeschehen schweben. Aus I 8 bis 2 I Kilometern Höhe stellen die Ballons Internetzugänge für Regionen zur Verfügung, die eingeschränkt oder gar nicht an das World Wide Web angeschlossen sind. Die mobilen Funkantennen werden in Gebieten eingesetzt, in denen sich der Ausbau terrestrischer Kommunikationsinfrastrukturen ökonomisch nicht lohnt oder in denen diese durch Naturkatastrophen vorübergehend blockiert oder beschädigt sind. Erfolgreiche Tests wurden erstmals 2013 in Neuseeland durchgeführt. Seit 2016 nutzt der Inselstaat Indonesien als erster Staat dauerhaft die Datenübertragung mit Ballons. 2017 hat Project Loon eine Lizenz erhalten, die vom Hurricane Maria zerstörten Kommunikationsinfrastrukturen Puerto Ricos vorübergehend mit Loons zu ersetzen. Seit 2019 wird mit Telkom Kenya daran gearbeitet, Bergdörfer mit 4G zu versorgen.

Da keine gültige Grenzziehung zwischen nationalem Luftraum und internationalem Weltraum existiert, ${ }^{8}$ bewegen sich die Internet-Ballons in der Stratosphäre in einem Raum, für den keine rechtliche Autorität zuständig ist. Staaten können zwar auf der Startseite von Project Loon das Kontaktformular für Regierungen ausfüllen - der Staat ist Kunde -, rechtliche Regelungen sind allerdings Verhandlungssache oder nicht gegeben. Die Stratosphäre ist weder Teil des im internationalen Recht als «airspace» bezeichneten Bereichs ziviler Luftfahrt noch des «outer space», welcher der internationalen Raumfahrt vorbehalten ist. ${ }^{9}$ Weder die Pariser Konvention von I9I9 noch die Chicagoer Konvention von 1944 und ebenso wenig die wissenschaftlich festgelegte Grenze von Atmosphäre und Weltraum, die sogenannte Kármán-Linie, sind internationaler Standard oder rechtlich bindend. ${ }^{10}$ Dass die Stratosphäre eine rechtliche Grauzone und damit eine politische Aushandlungszone ist, wird insbesondere dann offenbar, wenn die in großer Höhe unsichtbar agierenden Ballons ungeplant zum Vorschein kommen. So wurde in Kolumbien die Polizei gerufen, als ein vermeintliches UFO vom Himmel stürzte, das sich 
als Google-Ballon entpuppte. ${ }^{11}$ In Kenia berichteten Anwohner_innen über starke Kopfschmerzen, nachdem ein Ballon auf ein nahegelegenes Feld gestürzt war. ${ }^{12}$ Nach Testflügen in Neuseeland, Australien und Brasilien meldeten Wissenschaftler_innen der Global Union Against Radiation Deployment from Space gesundheitliche Bedenken an. ${ }^{13}$ Doch auch dort, wo die Abläufe technisch einwandfrei funktionieren, können die Ballons politische Reibungen erzeugen, für die der Verursacher - ein privates Unternehmen mit Sitz in den USA - nicht zwingend der Adressat ist, beispielsweise dann, wenn der Internetzugang aus der Luft in Staaten zur Verfügung gestellt wird, in denen er politisch reglementiert ist. ${ }^{14}$

Jenseits bekannter legislativer Prozesse formen die Ballon-Netzwerke die Stratosphäre zu einem jener «dynamic systems of space, information and power», die politische Formen schneller bilden, als Regierungen Gesetze erlassen können. ${ }^{15}$ Vergleichbar zu Infrastrukturprojekten in urbanen Räumen besetzen die Ballon-Netzwerke die Stratosphäre als extraterritorialen Raum, der als «medium of what might be called extrastatecraft $>{ }^{16}$ verstanden werden kann - ein Kofferwort, mit dem Easterling neue Formen des Regierens außerhalb staatlicher Kontrolle beschreibt. Googles Stratosphäre ist, vergleichbar mit Freihandelszonen, ein solcher extraterritorialer infrastructure space.

Die Marketingstrategie des Unternehmens stellt mit «Loon for All» und «Connect People Everywhere ${ }^{17}$ dabei konsequent eine Vermarktungsrhetorik aus Humanismus und Fortschrittlichkeit in die Tradition der Anfänge des Internets. Zu Beginn wurde das Projekt von Google X, der damaligen Forschungsabteilung des Konzerns, durchgeführt. X gibt an, seine Projekte voranzutreiben, um das Leben von Millionen, ja sogar Milliarden Menschen auf der ganzen Erde zu verbessern. Mit dieser Selbstdarstellung folgt X einem dominanten Mantra des Silicon Valley - making the world a better place. ${ }^{18}$ Seit Anfang 2017 ist Google X als eigenes Unternehmen namens X ebenso wie Google selbst dem Mutterkonzern Alphabet Inc. unterstellt. Mittlerweile ist Loon, das als eines der erfolgreichsten X-Projekte gilt, als eigenes Unternehmen ausgegliedert. X und Loon folgen noch einem weiteren Motto des Silicon Valley: mit innovativen Technologien funktionierende Märkte aufmischen. ${ }^{19}$ Die Begriffe $\mathrm{X}$ und Loon implizieren beide ein Faible für das Randständige und Unbekannte. $\mathrm{X}$ beschreibt sich selbst als «moonshot factory», als Zusammenschluss von Erfinder_innen und Unternehmer_innen, die das Risiko von Forschung mit der Geschwindigkeit eines Start-ups kombinieren würden. ${ }^{20}$ Der Name Loon wiederum spielt sowohl auf das ballförmige Luftfahrzeug als auch auf Verrücktheit an, balloon und loony.

«Shaping and managing the story», so Easterling, «is [...] an essential skill in infrastructure space. ${ }^{21}$ Je stärker infrastrukturierte Räume als «spatial products» hergestellt würden, umso mehr würden sie in die «irrational fictions of branding» eingepasst. Mit Fokus auf die «histories» solcher infrastructure spaces würden sich jene «stories» aufbrechen lassen, die von einer Organisation selbst
11 Google Balloon Mistaken for UFO as it Crashes in Colombia, in: BBC News, 14.3.2017, bbc.com/ news/world-latin-america-39265813 (6.6.2019).

12 Google's Project Loon Internet Balloon Crashes in Kenya, in: Beebom, 1.1.2018, beebom.com/googleproject-loon-internet-crashes-kenya (6.6.2019).

13 Vgl. Lizzy Keen: Scientists Raise Concerns about Google's Project Loon, in: Independent Australia, 7.6.2016, independentaustralia.net/ business/business-display/scientistsraise-concerns-about-googles-projectloon,9077 (6.6.2019).

$14 \mathrm{Vgl}$. Caswell: The Need for Vertical Delineation, 207.

15 Easterling: Extrastatecraft, 15. 16 Ebd.

17 Überschriften auf loon.com (13.5.2020).

18 Vgl. Parks, Graydon: ‘Connecting the Unconnected, 2.

19 Vgl. ebd.

$20 \mathrm{Vgl}$. Eintrag auf x.company (13.5.2020).

21 Easterling: Extrastatecraft, 22. Sie knüpft hier u. a. explizit an Bruno Latour an. 
22 Vgl. ebd., $168 \mathrm{f}$.

23 Dieter Zastrow: Entstehung und Ausbildung des französischen Vokabulars der Luftfahrt mit Fahrzeugen sleichter als Luft, (Ballon, Luftschiff) von den Anfängen bis 1910, Tübingen 1963, 39 .

$24 \mathrm{Vgl}$. Wilfrid de Fonvielle: Les ballons-sondes de MM. Hermite et Besançon et les ascensions internationales, Paris $1898,10 \mathrm{f}$. und $20 \mathrm{f}$. Die Konstrukteure knüpften an ein Wissen über das Verlorengehen an, das sich in der bemannten Ballonfahrt herausgebildet hatte, vgl. das Kapitel «Luftpost. Belagerung von Paris 1870-71» in: Hannah Zindel: Ballons. Medien und Techniken früher Luftfahrten, Paderborn 2020 (im Erscheinen), 69-89.

25 Vgl. John M. Bacon: The Dominion of the Air. The Story of Aerial Navigation, London 1902, 186. Zwar wurden die unbemannten Ballons nie für Polerkundungen eingesetzt, Hermite und Besançon berieten und trainierten jedoch den Wissenschaftler Salomon Auguste Andrée, der 1897 tatsächlich mit zwei Mitstreitern im Ballon zum Nordpol aufbrach. Trotz der Verbannung des Begriffs perdre aus der Luftfahrt ging dieser bekanntlich verloren. Zu Andrée vgl. Wolfgang Struck: Ingenjör Andrées luftfärd oder Die melancholischen Entdeckungen des Films, in: ders., Hansjörg Bay (Hg.): Literarische Entdeckungsreisen. Vorfahren - Nachfahrten - Revisionen, Wien u. a. 2012, 29-52.

26 Fonvielle: Les ballons-sondes, VIII.

27 Vgl. ebd., 10.

28 Vgl. Jean Mascart: L'étude de la haute atmosphère et les travaux de Léon Teisserenc de Bort, in: La Nature, Nr. 208o, 5. April 1913, 296-300.

29 John L. Dubois, Robert P. Multhauf, Charles A. Ziegler: The Invention and Development of the Radiosonde. With a Catalog of UpperAtmospheric Telemetering Probes in the National Museum of American History, Washington D.C. 2002, 8. $\mathrm{zu}$ «influential fiction» aufbereitet würden, um das, was sie sagen, von dem zu trennen, was sie machen. ${ }^{22}$ Die Marke Loon verspricht Zugang zu Bildung, Gesundheit und Arbeitsplätzen. Sie spielt mit technischen und wissenschaftlichen Fortschritts- und Expansionsphantasmen. Ballons, so verdeutlichen im Folgenden Technik- und Wissenschaftsgeschichten unbemannter Ballons und Simultanaufstiege, dienen Google nicht nur als Transportmittel besonderer Technologien, sondern auch als Träger eines spezifischen Images, das sich in diese Markenbildung einpasst.

\section{Techniken unbemannter Ballons: «ballon perdu», «ballon-sonde», «loon»}

Fahrten ohne Aeronaut_innen an Bord wurden in den Anfangsjahren der Ballonfahrt auch als «à ballon perdu $»^{23}$ bezeichnet - im verlorenen Ballon. I 892 statteten der Ballonist Gustave Hermite und der Wissenschaftler Georges Besançon ballons perdus mit selbstschreibenden Instrumenten, reißenden Papierhüllen und Postkarten an langsam abbrennenden Zündschnüren aus (Abb. I). ${ }^{24}$ Aufschreiben, Landen und Wiederfinden wurden mittels dieser zusätzlichen Ausrüstung teilautomatisiert, sodass die unbemannten Ballons den Himmel ohne notierende Forscher_innen und steuernde Ballonkapitän_innen vermessen konnten. Die beiden Konstrukteure hatten mit ihren unbemannten Luftfahrzeugen anfänglich versucht, die ${ }^{8} 86_{3}$ in einer Publikation von Delaville Dedreux entwickelte Idee einer Exkursion zum Nordpol im Ballon umzusetzen..$^{25}$ Hierfür verwendeten sie nicht die gängige Bezeichnung à ballon perdu, sondern nannten ihre Luftkugeln «ballons-sondes» ${ }^{26}$ Perdre - verlieren, leck sein, undicht sein - ersetzten sie durch sonder - sondieren, ausloten, vermessen. Da ihnen die Finanzierung für die Polunternehmung fehlte, konzentrierten sich die beiden Franzosen vorerst auf Versuche zur Vermessung hoher Himmelsregionen. ${ }^{27}$ Gegenüber bemannten Ballons, Fesselballons und Drachen hatten unbemannte Ballons nämlich den Vorteil, dass sie auch Höhen von über acht Kilometern erreichen konnten. Hermite und Besançon stellten ihre ballons-sondes I896 in Paris auf einer der ersten internationalen meteorologischen Konferenzen vor. Im Anschluss wurden die Ballon-Sonden systematisch für geophysikalische Forschungen in der Atmosphäre eingesetzt. So unterschied etwa der französische Meteorologe Léon-Philippe Teisserenc de Bort nach Aufstiegen von ballons-sondes 1902 die Atmosphäre in Tropo- und Stratosphäre. ${ }^{28}$ Als Instrumente der Wettervorhersage eigneten sich die unbemannten mobilen Observatorien allerdings nicht, da die Verarbeitung der mit ihnen aufgezeichneten Daten - falls sie überhaupt gefunden wurden - meist nach mehreren Tagen oder auch Wochen erfolgte. ${ }^{29}$

Wettervorhersagen waren es allerdings, die private und staatliche Geldgeber_innen interessierten. Mit dem Ersten Weltkrieg hatte sich gezeigt, dass eine einsatzfähige Luftwaffe ebenso auf verlässliche Wetterdaten aus verschiedenen Höhen angewiesen war wie auf Artilleriefeuer und chemische 
Kriegsführung. ${ }^{30}$ Auch in der zivilen Luftfahrt zum Transport von Personen und Waren sowie in der Landwirtschaft bestand ein großes Interesse an zuverlässigen Wettervorhersagen. ${ }^{31}$ Finanziert und vorangetrieben wurden Versuche zur drahtlosen und damit zeitnahen Übertragung der mit unbemannten Ballons registrierten Daten daher vor allem im und nach dem Ersten Weltkrieg von den Regierungen in Deutschland, Frankreich, der Sowjetunion und den Vereinigten

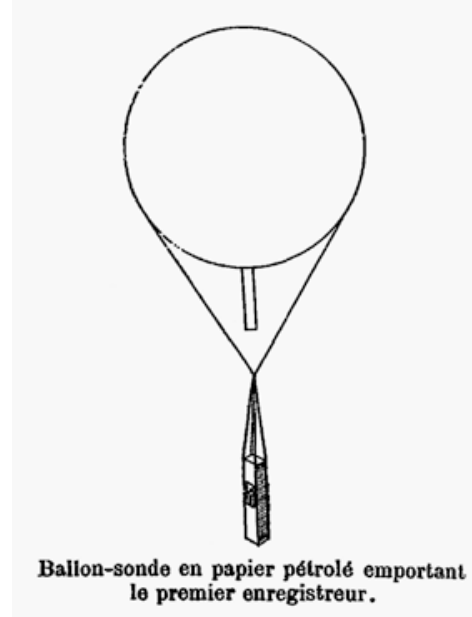
Staaten. Wie von Technikhistorikern des National Museum of American History gut erforscht, galt es für den Schritt von der Ballonsonde zum Wetterballon Radiotechnik an die Umwelt von Ballons anzupassen. ${ }^{32}$ Die Entwicklung kleiner leichter Transmitter, die auch von Ballons getragen werden konnten, ermöglichte Ende der I92oer Jahre schließlich eine drahtlose Datenübertragung von Ballons zu Bodenstationen mittels Radio; Wetterballons werden daher auch als Radiosonden bezeichnet (Abb. 2). ${ }^{33}$ I 940 waren diese technisch so weit stabilisiert, dass sie in Massenproduktion gingen und die bis dahin gebräuchlichen Ballonsonden von den nationalen Wetterdiensten offiziell durch Radiosonden ersetzt wurden. ${ }^{34}$ Dank Cellophanpapierhüllen konnten die Radiosonden zudem deutlich höher steigen, sodass sie auch als Kundschafterinnen vor Raketenstarts eingesetzt sowie zu weiteren geophysikalischen Forschungen an den Rändern der Atmosphäre verwendet werden konnten. ${ }^{35}$ Entwicklungen in der gleichzeitigen mehrkanaligen Übertragung einzelner von Sensoren gemessener Daten während des Zweiten Weltkriegs wurden zudem für Entwicklungen in der Luftfahrt und der analogen Telemetrie zentral. ${ }^{36}$

Vor dem Hintergrund dieses technikhistorisch informierten Exkurses über unbemannte Ballons lässt sich festhalten, dass diese sich vom ballon perdu über den ballon-sonde zur Radiosonde und von unlenkbaren Luftfahrzeugen der Mechanik um I80o über teilautomatisierte mobile Observatorien der Meteorologie um I 900 zu Instrumenten der Wettervorhersage für Militär, Landwirtschaft sowie Luft- und Raumfahrt um I940 entwickelten. Im Zuge dessen wandelte sich die Stratosphäre nachrichtentechnisch von einem Tohuwabohu fliegender Flaschenposten zu einer Routenkarte störungsfrei zirkulierender Datenpakete. Nach ballonbegeisterten und geowissenschaftlich interessierten Einzelpersonen, die beim transnationalen Rennen um die Entdeckung des Nordpols mitmischen wollten, waren es die am Aufbau einer grenzübergreifend operierenden Erforschung der Atmosphäre beteiligten Meteorolog_innen, welche die

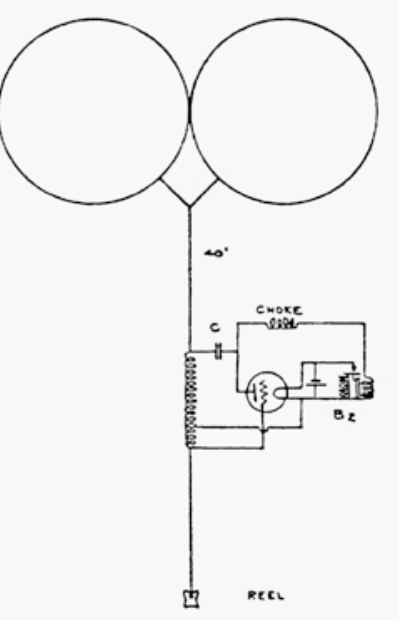

Abb. 1 Unbemannter Ballon von Hermite und Besançon, Paris 1892

Abb. 2 Radiosonde von William Blair, $1923 / 24$

30 Vgl. ebd., 26.

31 Vgl. Robert Marc Friedman: Appropriating the Weather. Vilhelm Bjerknes and the Construction of a Modern Meteorology, Ithaka 1989, 115. $32 \mathrm{Vgl}$. Dubois u. a.: The Invention, 25.

33 vgl. ebd., 14.

34 Vgl. ebd., 59 .

35 Vgl. ebd., 66.

36 vgl. ebd., 65 f. 


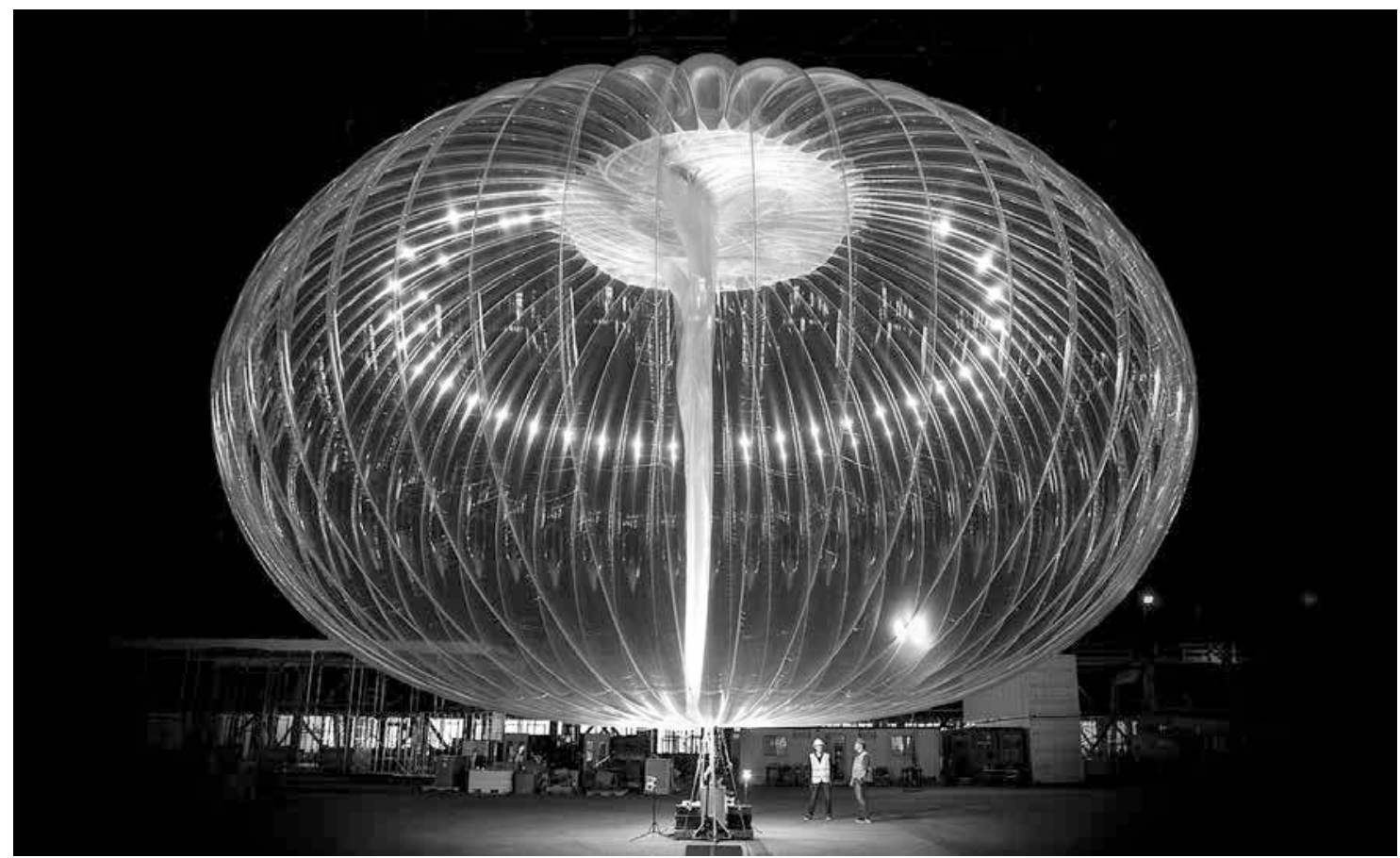

Abb. 3 Entfalteter Loon im Test

37 Vgl. «Loon System», loon.com/ technology (12.6.2019).

38 Vgl. ebd.

39 Max Engel: Google's Project Loon Hovers Over the Satellite Industry, in: VIA Satellite, Bd. 28 , Nr. 8, 2013, www.satellitetoday.com/ telecom/2013/08/01/googles-projectloon-hovers-over-the-satellite-industry| $(3.7 \cdot 2020)$
Versuche mit den unbemannten Registrierballons weiterführten; ihnen wiederum folgten an staatlich finanzierten Wissenschaftseinrichtungen angestellte Nachrichtentechniker_innen.

Mit den von einem Privatunternehmen entwickelten Loons setzt sich die Anpassung von Ballon- und Nachrichtentechniken an die räumlichen Bedingungen der Stratosphäre fort (Abb. 3). Um in den klimatisch extremen Bedingungen bestehen zu können, müssen Internet-Ballons und die von ihnen mitgeführte Ausstattung deutlich länger als Radiosonden Temperaturen bis zu minus 90 Grad Celsius, Windgeschwindigkeiten bis zu roo Stundenkilometer und intensive UV-Strahlung überdauern. ${ }^{37}$ Aus drei Millimeter starkem Polyethylen hergestellt, sollen sie mindestens io Tage lang halten. Solarpanels dienen tagsüber der Energieversorgung und laden eine Batterie für den nächtlichen Betrieb. ${ }^{38}$

Loons sind im Unterschied zu Wetterballons nicht nur schwebende Observatorien, sondern auch fliegende Mobilfunkantennen. Die initiale Idee war es 2013, mit den Ballons 3 G-Breitbandinternet für LTE-fähige Endgeräte, also z. B. Smartphones anzubieten. Mobil sollte dieses für Regionen zur Verfügung gestellt werden, die weder mit Satelliten versorgt werden können, welche Rundfunkangebote über einen Transmitter zu vielen Receivern übertragen, noch mit Satelliten, die Zwei-Wege-Breitband-Angebote an feste Orte mit einer entsprechenden Antenne liefern. ${ }^{39}$ Vom ursprünglichen Plan, Frequenzen im Radiospektrum zu kaufen, hatte Google Abstand genommen; die 
Ballons können sich stattdessen mittels der nächstgelegenen Mobilfunkmasten auf der Erde mit bestehenden Telekommunikations-Netzwerken verlinken. ${ }^{40}$ Aktuell übertragen Antennen an Bord Datenpakete von Mobilfunkmasten auf der Erde über ein Mesh-Netzwerk aus Ballons zu LTE-fähigen Endgeräten auf dem Boden. Untereinander sind die Ballons über Laser mit optischen Signalen verbunden. ${ }^{41}$ Zentral für die Funktion der Loons als Observatorium und Antenne ist die Gruppierung mehrerer Ballons zu Clustern, die im Verhältnis zueinander sowie zu den Mobilfunkmasten und Endgeräten auf dem Boden positioniert werden.

Aus medienwissenschaftlicher Perspektive verändern sich unbemannte Ballons mit dem Schritt von der Radiosonde zum Loon zu 〈smarten〉 Objekten, die vernetzt, ubiquitär und pervasiv auftreten..$^{42}$ Als doppelte «everyware ${ }^{43}$ verlegen sie Informationsverarbeitung nicht nur aus den Geräten der Nutzer_innen heraus, sondern in die selbst in Bewegung befindliche und für viele Objekte und Signale durchlässige Luft. «Embedded with sensors and digital media» verwandelt sich die Stratosphäre von einem sondierten zu einem sondierenden Raum; die Loon-Netzwerke nutzen die materiellen Eigenschaften der Luft als «information technology» und «operating system». ${ }^{44} \mathrm{Um}$ die skizzierten Entwicklungen in der Sensor- und Telemetrie-Entwicklung an Schnittstellen von Ballonfahrt und Nachrichtentechnik zu einer großskaligen schwebenden Kommunikationsinfrastruktur auszubauen, verwendet Google quantitative Verfahren der Datenerhebung und numerische der Datenverarbeitung, die sich, wie im Folgenden nachgezeichnet wird, nicht zuletzt an Schnittstellen von Meteorologie und Ballonfahrt herausgebildet haben.

\section{Simultanaufstiegswissen: Luftstrommuster, Vorhersagefabrik, Windschach}

I896 wurden in Paris bei dem Treffen der Direktoren der meteorologischen Institute verschiedener Länder nicht nur die ballons-sondes vorgestellt, sondern auch die Internationale Kommission für wissenschaftliche Luftfahrt eingesetzt, um die bis dahin an Einzelpersonen hängende Beobachtung der Atmosphäre über die Ländergrenzen hinweg zu organisieren. ${ }^{45}$ Bei dem zweiten Treffen der Kommission I900 wurde beschlossen, sogenannte Simultanaufstiege mit Ballons als offizielles Beobachtungswerkzeug einzusetzen. ${ }^{46}$ Die gleichzeitigen und europaweiten Messungen sollten es ermöglichen, statt lokaler Wetterphänomene globale «Witterungsgebilde» zu studieren. ${ }^{47}$ Während Ballontrajektorien einzelnen Luftströmungen entsprachen, sollten Ballon-Cluster es erlauben, Luftstrommuster zu bestimmen - ein Ansatz, mit dem auch Google operiert.

Praktisch sammelten sich die während der Simultanaufstiege erhobenen Daten in Form von Tabellen allerdings in so großer Menge bei dem Vorsitzenden der Kommission in Straßburg, dass sie vorerst niemand auszuwerten vermochte. ${ }^{48}$ Einzig das synoptische Verfahren - z. B. das Zeichnen von
40 Vgl. Tom Simonite: Project Loon. Billions of People Could Get Online for the First Time Thanks to Helium Balloons, in: Technology Review, Bd. 118, Nr. 2, 2015, 40-45.

41 Über die technischen Verfahren gibt X kaum Details bekannt. Ende 2017 hat Alphabet Inc. mit der indischen Region Andhra Pradesh ein Abkommen geschlossen, diese mittels eines Spin-offs aus dem Loon-Projekt mit Internet zu versorgen. Tests wurden begonnen, um mithilfe von auf Dächern installierten Boxen Datenpakete von bis zu 20 Gigabit pro Sekunde mittels Lichtstrahlen zu übertragen. Vgl. Paresh Dave: Alphabet's X Sells New Wireless Internet Tech to Indian State, in: Reuters, 15.12.2017, reuters.com/article/us-alphabet-india/ alphabets-x-sells-new-wireless-internettech-to-indian-state-idUSKBN1 E8 3 BQ (19.6.2019).

42 Vgl. Adam Greenfield: Everyware. The Dawning Age of Ubiquitous Computing, Berkeley 2006.

43 Vgl. ebd.

44 Easterling: Extrastatecraft, $13 \mathrm{f}$.

45 Vgl. Sabine Höhler: Luftfahrtforschung und Luftfahrtmythos. Wissenschaftliche Ballonfahrt in Deutschland 1880-1910, Frankfurt/M. 2001, 286-289. Die Kommission wurde auch Internationale Aeronautische Kommission genannt.

46 Vgl. ebd., 286-289.

47 Julius Maurer: Die meteorologischen Ergebnisse, in: Albert Heim, Julius Maurer, Eduard Spelterini: Die Fahrt der "Wega" über Alpen und Jura am 3. Oktober 1898, Basel 1899, 82-125, hier 119.

481903 wurden die gesammelten Daten in Form der Veröffentlichungen der Internationalen Kommission für wissenschaftliche Luftschiffahrt herausgegeben, damit sie wenigstens zugänglich gemacht werden konnten. 


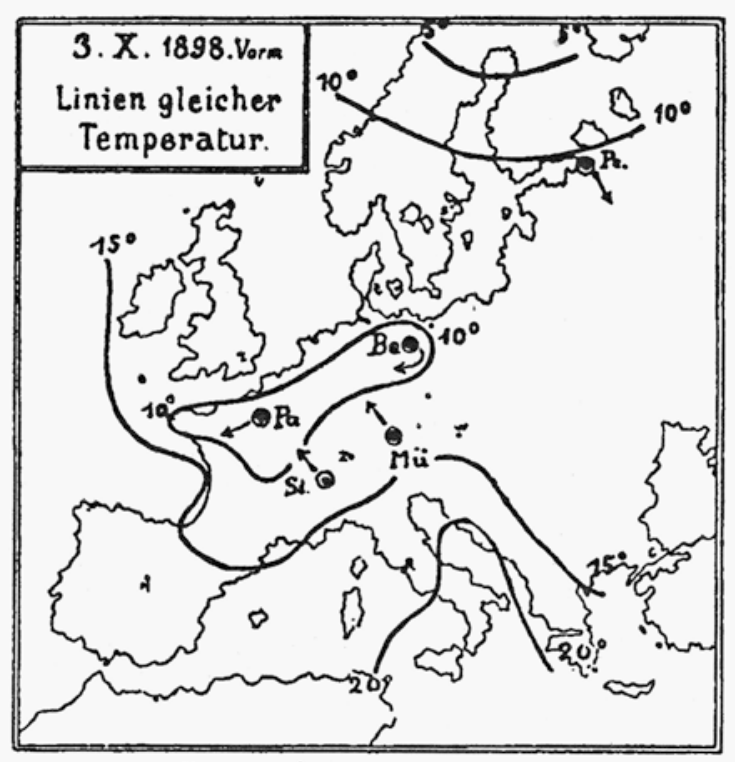

Pfeile - Fugrichtung der Bullons

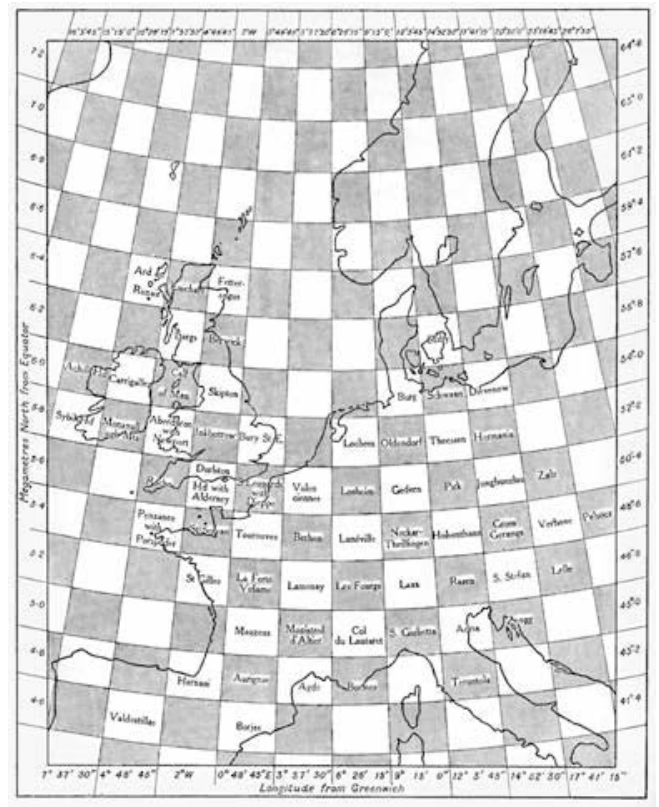

Abb. 4 Europakarte mit Aufstiegsorten der Simultanaufstiege zur synoptischen Wettervorhersage, 1898

Abb. 5 Europakarte von Lewis Fry Richardson zur numerischen Wettervorhersage, 1922
49 Vgl. Friedman: Appropriating the Weather. Synoptische Meteorologie wurde mangels anderer Datenauswertungsverfahren bis in die $1960 e r$ Jahre hinein praktiziert. So ließ sich die Bewegung von Wetterfronten grafisch nachvollziehen.

50 Lewis Fry Richardson: Weather Prediction by Numerical Process, London 1922, 2.

51 Unbemannte Ballons wurden im 19. Jahrhundert vor allem als sogenannte Pilotballons eingesetzt, um die Windrichtung vor bemannten Aufstiegen zu prüfen.
Linien gleicher Temperatur - stand zur Auswertung der notierten Messwerte zur Verfügung (Abb. 4). Da die synoptische Methode als langsam und ungenau galt, schlug I904 der Meteorologe Vilhelm Bjerknes vor, dass sie ersetzt werden solle durch eine theoretische Auswertung mit Gleichungen, die auf den physikalischen Gesetzen der Atmosphäre basierten. ${ }^{49}$ Wie in der Geschichte der Meteorologie und des Computers hinlänglich beschrieben, wurde eine numerische Wettervorhersage, wie Bjerknes sie forderte, I922 vom Engländer Lewis Fry Richardson in seinem Buch Weather Prediction by Numerical Process veröffentlicht (Abb. 5). Um seine Formeln zu überprüfen - das ist ein in der Forschungsliteratur meist nur peripher beleuchteter Punkt -, hatte Richardson I9I 7 ein besonderes Beispiel durchgerechnet: eine nachträgliche Wettervorhersage für den 20. Mai ı 9ıо, für den aufgrund der internationalen Simultanaufstiege mit Ballons der umfangreichste Datensatz vorlag, bestehend aus Messwerten terrestrischer Wetterstationen und unbemannter mobiler Ballonobservatorien.

This region and instant were chosen because the observations form the most complete set known to me at the time of writing. [...] Unfortunately this <forecast> is spoilt by errors in the initial data for winds. These errors appear to arise mainly from the irregular distribution of pilot balloon stations, and from their too small number. ${ }^{\mathbf{5 0}}$

Unbemannte Ballons - hier als pilot balloons bezeichnet ${ }^{51}$ - und Simultanaufstiege können mit Richardson nicht nur als Instrumente der Datenerzeugung, sondern auch als zentrale Glieder in Ketten der elektronischen Datenverarbeitung betrachtet werden. Wie der Meteorologe schreibt, kam er wegen eines 
Rechenfehlers zwar nicht auf die Vergleichswerte, die ihm für den Maitag vorlagen, aber er bewies, dass eine numerische Vorhersage grundsätzlich möglich war. Um die von ihm benötigte Rechenzeit von sechs Wochen annähernd auf Echtzeit zu reduzieren, imaginierte Richardson mangels maschineller Computer eine Vorhersagefabrik mit 64.000 menschlichen Computern. Diese Fabrik ähnelt jener «vast machine», die John Ruskin I839 in einer Rede vor der Londoner Meteorological Society entworfen hatte und die Paul Edwards in seinem die «vast machine» im Titel tragenden Buch so prominent besprochen hat: ${ }^{52}$ eine den Globus umspannende Wettervorhersagemaschine, in welcher die Atmosphäre bis ins kleinste Detail aufzuzeichnen und vorherzusehen ist. In einer Zeichnung von Richardsons Fabrik interpretiert der Künstler François Schuiten diese als Panoptikum (Abb. 6).

Die Meteorologie, so die Wissenschaftshistorikerin Sabine Höhler, wurde insbesondere unter Beteiligung der Aerologie, also der Höhenwetterkunde mit unbemannten Ballons, von einer empirisch-beobachtenden in eine theoretisch-rechnende Wissenschaft umstrukturiert. Die internationale Zusammenarbeit der Wissenschaftler_innen im Rahmen der Simultanaufstiege habe zur Etablierung einer Physik der Atmosphäre beigetragen und langfristig die Voraussetzungen für die systematische Vermessung und Kartierung der freien Atmosphäre geschaffen. ${ }^{53}$ Heutige Vorhersagemodelle basieren auf numerischen Methoden, die mit Supercomputern durchgerechnet und alle sechs Stunden mit neuen Messdaten, überwiegend von Satelliten, gespeist werden. ${ }^{54}$ Durch den zunehmenden Ausbau an Wetterstationen und die expandierende Rechenleistung nimmt die Genauigkeit dieser Modelle zwar zu, allerdings bleibt weiterhin das Problem der mathematischen Modellierung bestehen, das auch schon Richardson Schwierigkeiten bereitete: Durch leichte Abweichungen in den Eingabedaten können große Abweichungen in den Ausgabedaten erzeugt werden..$^{55}$

Die Bestimmung von Windmustern in der Stratosphäre durch Project Loon rückt einen kleinen Schritt näher an den meteorologischen Traum von Ruskin und Richardson heran. Zur Navigation seiner Ballons ist Google darauf angewiesen, die Bewegungen der Stratosphäre vorhersehen zu können. «You can think of this as very detailed sailing, almost like a game of chess with the winds», ${ }^{\mathbf{5 6}}$ so Astro Teller, Leiter von Google X. Für dieses Windschachspiel arbeitet Google mit Gauß-Prozessen, einer Vorhersagemethodik, die es erlaubt, auf Basis von vergleichsweise wenig Daten mit hohen Unsicherheiten umzugehen. ${ }^{57}$ Mit dieser Form des Machine-Learning lassen sich nicht nur Muster in der Stratosphäre erkennen; sie agiert auch in der realen Welt außerhalb des Computers. ${ }^{58}$ Denn anders als die nationalen Wetterdienste nutzt Google Ballon-Cluster nicht nur zum Aufzeichnen, Verarbeiten und Speichern meteorologischer Messdaten, sondern auch dazu, einen Maschinenlernalgorithmus zu trainieren, der auf Basis früherer und aktueller Flugwege zukünftige Routen bestimmt. Loons lassen sich steuern.
52 Vgl. Paul N. Edwards: A Vast Machine. Computer Models, Climate Data, and the Politics of Global Warming, Cambridge 2010, 8 . 53 Vgl. ebd., $284 \mathrm{f}$.

54 Vgl. Gabriele Gramelsberger: Computerexperimente. Zum Wandel der Wissenschaft im Zeitalter des Computers, Bielefeld 2010.

$55 \mathrm{Vgl}$. ebd.

56 Zit. n. Cade Metz: Machine Learning Invades the Real World on Internet Balloons, in: Wired, 17.2.2017, www.wired.com/2017/02| machine-learning-drifting-real-worldinternet-balloons (22.5.2020).

57 Ebd.

58 Vgl. ebd. 


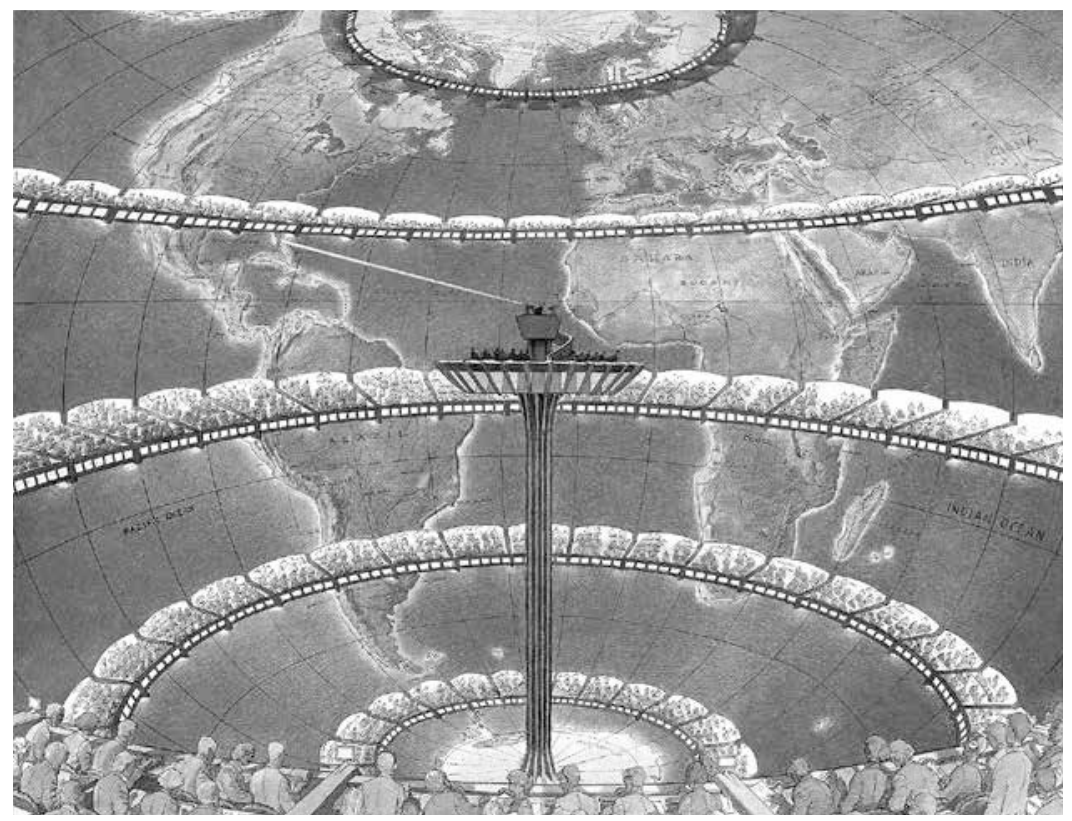

Abb. 6 Richardsons Vorhersagefabrik, illustriert von François Schuiten, o.J. (Orig. in Farbe)
59 Geprägt hatte die Prinzipien und Lager der Luftfahrt Leichterbzw. Schwerer-als-Luft 1670 der Jesuit Francesco Lana in seiner Beschreibung eines Luftbootes, vgl. Zastrow: Entstehung und Ausbildung des französischen Vokabulars der Luftfahrt, 30.

\section{Ballonsteuerungen: ohne «Maschienerie» und mit «decision-making algorithms»}

Ein zentrales Problem der Ballonfahrt besteht darin, dass Ballons nicht zu steuern sind. Sie treiben mit dem Wind. Von den ersten Aufstiegen mit Heißluftund Gasballons ${ }_{1} 78_{3}$ bis zu heutigen Einsätzen von Passagier- und Wetterballons lassen sich einzig das Höhersteigen oder Hinabsinken der Luftfahrzeuge kontrollieren. Dazu wird wahlweise die Luft in der Hülle erhitzt und abgekühlt oder Ballast abgeworfen und ein Ventil geöffnet. Die Frage, ob und wie sich das Luftfahrzeug horizontal navigieren lässt, war seit den ersten Jahren der Ballonfahrt an die Frage gekoppelt, wozu Aerostaten überhaupt zu gebrauchen waren. Den Anhänger_innen des Leichter-als-Luft-Prinzips gelang nach einigen Fehlschlägen mit Schrauben, Rudern und Segeln gegen Ende des I9. Jahrhunderts die Konstruktion von Luftschiffen (gewissermaßen motorisierte Ballons). Die Befürworter_innen des Schwerer-als-Luft-Prinzips hatten mit dem Bau von Flugzeugen Erfolg. ${ }^{59}$

Für eine gänzlich andere Art der Steuerung von Ballons machte sich Heinrich von Kleist stark, die er I8 10 in den Berliner Abendblättern darlegte. Sein Zugang konzipierte ebenso wie der von Google das Steuern nicht vom Objekt, sondern von dem ihn umgebenden Raum her.

Der Wachstuchfabrikant Hr. Claudius will [...] mit dem Ballon [...] in die Luft gehen, und denselben, vermittelst einer Maschine, unabhängig vom Wind, nach einer bestimmten Richtung hinbewegen. Dies Unternehmen scheint befremdend, da die Kunst, den Ballon, auf ganz leichte und naturgemäße Weise, ohne alle Maschienerie, 
zu bewegen, schon erfunden ist. Denn da in der Luft alle nur mögliche Strömungen (Winde) übereinander liegen: so braucht der Aeronaut nur vermittelst perpendikularer [d. i. senkrechter] Bewegungen, den Luftstrom aufzusuchen, der ihn nach seinem Ziel führt. ${ }^{60}$

Kleist schlug vor, Ballons durch das gezielte Ansteuern von Luftströmungen zu navigieren. Sein Ansatz verschob die Lenkbarkeitsfrage vom Gebiet der Mechanik in das der Meteorologie. ${ }^{61}$ Zwar blieben Luftströme zu unberechenbar, um Ballons gezielt von A nach B zu steuern. Doch pointiert Kleists Vorschlag die besonderen ballistischen Eigenschaften des Leichter-als-Luft-Fahrzeugs. Für Ballons als «Medien der Bewegung» ${ }^{62}$ gilt nicht, was Paul Virilio für Autos, Eisenbahnen und Flugzeuge herausgearbeitet hat. Das Luftfahrzeug schießt nicht «mit der Gewalt eines Projektils» und «unberührt vom durchquerten Raum ${ }^{63}$ durch die Landschaft. Ballons sind Medien der Bewegung dessen, was sie bewegt. Das Gasgemisch im Inneren der Ballonhülle verhält sich bei vertikalen und horizontalen Bewegungen in ständigem Streben nach einem Equilibrium zu dem außerhalb der Hülle. Ballons zu steuern bedeutet, dieses Gleichgewicht zu regulieren; im Grunde genommen balancieren Ballons durch den Himmel.

Diese metonymische Beziehung des Luftfahrzeugs zu dem ihn umgebenden Raum macht sich Google mit dem Schritt vom Ballon zum Loon zunutze, indem es sie in eine reziproke verwandelt. Im Rennen um die passende Technologie für das Internet für alle setzt das Unternehmen auf eine modifizierte Version des besonderen Wechselverhältnisses von Ballons und Atmosphäre. Die für vielerlei Anwendungen ungünstige Eigenschaft von Leichter-als-LuftFahrzeugen, mit dem Wind zu treiben, wird von Google ins Positive gewendet, indem der Wind als energieeffizienter Antrieb genutzt wird. Um die Ballons gezielt zu einer Luftströmung zu bringen, die sie zur richtigen Position leitet, hat Google eine besondere Technik entwickelt.

In the stratosphere winds are stratified, and each layer of wind varies in speed and direction. To get balloons to where they need to go, Project Loon uses predictive models of the winds and decision-making algorithms to move each balloon up or down into a layer of wind blowing in the right direction. By moving with the wind, the balloons can be arranged to provide coverage where it's needed. ${ }^{64}$

Die Google-Ballons navigieren, indem sie sich nach oben oder unten in den jeweils passenden Luftstrom bewegen. ${ }^{65}$ Das gezielte Ansteuern ausgewählter Luftströme wird von einer Software in einem Google-Datencenter koordiniert, welche die Windvorhersagen der U.S. National Oceanic and Atmospheric Administration in ein Modell von stratosphärischen Luftströmen einarbeitet. Kombiniert werden diese mit aktuellen und früher erhobenen Daten, welche die Stratosphärenballons auf Millionen von Kilometern an Testflügen gesammelt haben. ${ }^{66}$ So lassen sich die Flugrouten der Ballons, die keinen eigenen Antrieb, wohl aber regulierbaren Auftrieb haben, entlang der in der Stratosphäre übereinanderliegenden Luftströme (strata) bestimmen. Auf diese Weise kann

\footnotetext{
60 Heinrich von Kleist: Schreiben aus Berlin, in: Sämtliche Werke. Brandenburger (Berliner) Ausgabe, Bd. I/1-IV/3, hg. v. Roland Reuß, Peter Staengle, Frankfurt/M., Basel 1888-2002, 65-66, Z. 25-30. $61 \mathrm{Vgl}$. Roland Borgards: Experimentelle Aeronautik. Chemie, Meteorologie und Kleists Luftschiffkunst in den ‘Berliner Abendblättern, in: Kleist-Jahrbuch 2005, hg. v. Günter Blamberger u. a., Stuttgart, Weimar 2005, 142-161.

62 Paul Virilio: Das Fahrzeug, Berlin 1978, 21.

63 Wolfgang Schivelbusch: Geschichte der Eisenbahnreise. Zur Industrialisierung von Raum und Zeit im 19. Jahrhundert, Frankfurt/M. 2011 [1977], 16 und 40.

64 "Navigating the Winds", loon.com/technology (24.4.2018).

65 Vgl. Simonite: Project Loon, 44. 66 Vgl. ebd.
} 

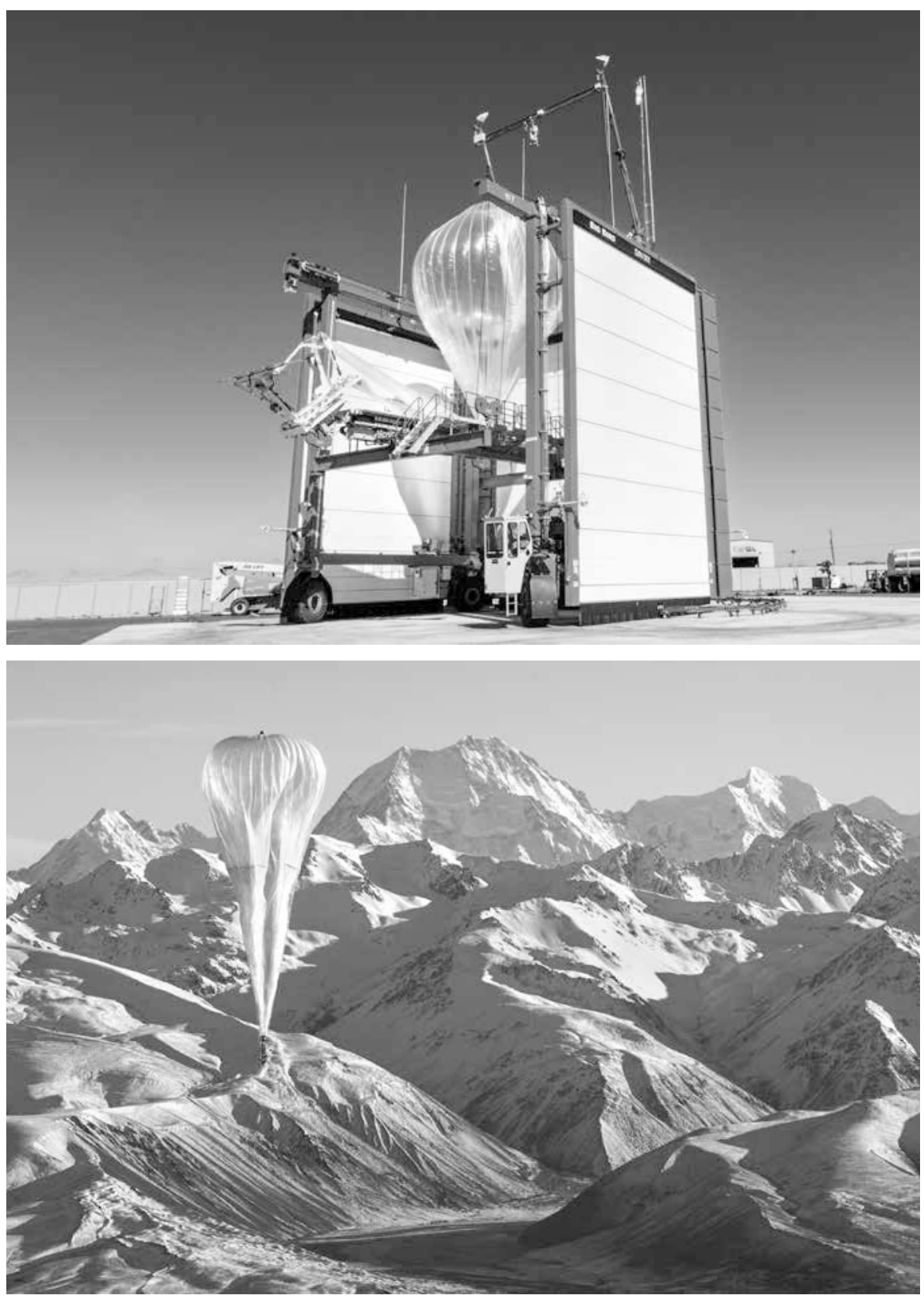

Abb. 7 Ein Autolauncher speist neue Loons in das Netzwerk ein.

Abb. 8 Ein Internet-Ballon der Firma Google 
ein Cluster von Google-Ballons von jedem beliebigen Punkt aus ein Gebiet erreichen, das nicht flächendeckend mit Internet versorgt ist und zwecks einer stabilen Konnektivität mehrere Wochen über diesem «kreisen〉.

Ebenso werden mit dem Schritt vom Ballon zum Loon die Apparaturen und Praktiken des vertikalen Aufsteigens, Steuerns und Landens überarbeitet. An weltweit verteilten Startstationen sollen Autolauncher - große mobile Gerüste, die automatisiert einen Ballon befüllen und steigen lassen - in der Lage sein, alle 30 Minuten einen neuen Loon in das Netzwerk einzuspeisen (Abb. 7). ${ }^{67}$ Mittels einer Konstruktion, die stark an die geheime Maschine aus Jules Vernes Roman Cinq Semaines en Ballon erinnert, können die Ballons steigen oder sinken. ${ }^{68}$ Dazu wird mit einer eingebauten Pumpe Luft eingepumpt oder abgesaugt, sodass sich die Dichte des Heliums im Inneren der Hülle verändert. ${ }^{69}$ Wenn ein Loon zur Landung gebracht werden soll, wird er von einem Google-Kontrollzentrum in Kooperation mit lokalen Flughäfen an einen abgelegenen, aber gut erreichbaren Ort geleitet. Das Helium wird mittels eines Ventils herausgelassen und das Equipment kehrt gesichert durch einen Fallschirm zurück zur Erde. Sogenannte recovery teams sammeln die Loons zum Recyceln auf.

Loons lassen sich aus medienwissenschaftlicher Perspektive - um einen Begriff von John Durham Peters aufzugreifen - als «sky media» ${ }^{70}$ in doppelter Hinsicht beschreiben. Als sendende Observatorien funktionieren sie ähnlich wie Wetterballons als Teil einer «climate knowledge infrastructure», ${ }^{71}$ die Daten über Wetter und Klima erhebt, speichert und verarbeitet. Als observierende Antennen lassen sie sich im Anschluss an Florian Sprenger als technologische Objekte fassen, die sich nicht losgelöst von ihren technisierten Umgebungen betrachten lassen und «durch ihre Kapazitäten der Vermittlung, Lokalisierung, Sensorik und Datensammlung [...] die von ihnen registrierten, überwachten und kontrollierten Räume zu environments auf[bereiten], in denen die Orte von Objekten durch ihre Umgebungsrelationen bestimmt werden». ${ }^{72}$ Die Stratosphäre wird von den Loons in situ mittels Sensoren vermessen und die Messergebnisse werden in den Datenzentren, in denen die entsprechende Rechenleistung zur Verfügung steht, verarbeitet, gespeichert und abgerufen. Mit dem Schritt von der Radiosonde zum Loon wandelt sich die metonymische Beziehung des Leichter-als-Luft-Fahrzeugs und des ihn umgebenden Luftraums in eine reziproke. Die Ballongondel und das in der Ballonhülle eingeschlossene Stück Atmosphäre werden verwoben mit weiteren Medien der Stratosphäre: einem komplexen technischen Geflecht aus Sensoren, Datenzentren, Vorhersagemodellen, Entscheidungsalgorithmen, Autolaunchern, Pumpvorrichtungen und Kontrollzentren. Project Loon verschachtelt Ballon und Ballonmodell, physikalischen und mathematischen Luftraum. Es unterläuft Unterscheidungen in vermeintlich natürliche und technische Räume. Für Project Loon ist die Stratosphäre kein thermodynamischer Raum zufälliger Luftströmungen und Turbulenzen, sondern ein mathematisch modellierter und im Computer simulierter Raum, keine unberechenbare Natur, sondern ein berechenbares environment.
67 Vgl. Caswell: The Need for Vertical Delineation, 208.

68 Vgl. das Kapitel «Kartenreise. Cinq Semaines en Ballon 1863" in: Zindel: Ballons, 45-67. 69 vgl. ebd.

70 Vgl. John Durham Peters: The Marvelous Clouds. Toward a Philosophy of Elemental Media, Chicago 2015, 253.

71 Edwards: Vast Machine, 8.

72 Florian Sprenger: Epistemologien des Umgebens. Zur Geschichte, Ökologie und Biopolitik künstlicher environments, Bielefeld 2019, 19. Sprenger schließt hier explizit an Erich Hörl an. 


\section{Schlussbemerkungen}

Der technikhistorisch informierte Blick auf Geschichten unbemannter Ballons ab den I 89oer Jahren hat gezeigt, wie Praktiken des Verfolgens bewegter Objekte im dreidimensionalen Raum und Technologien der drahtlosen Datenübertragung über weite Distanzen erprobt und standardisiert wurden. Der wissenshistorisch informierte Blick auf Simultanaufstiege ab I90o hat verdeutlicht, wie sich lose wissenschaftliche Kooperationen innerhalb und außerhalb wissenschaftlicher Einrichtungen verfestigten und institutionalisierten und wie aerologische Forschungen die Meteorologie maßgeblich zu einer statistisch-rechnenden Wissenschaft umstrukturierten. Googles Ballon-Netzwerke knüpfen an diese Technologien und Praktiken an und entwickeln sie weiter. Zudem setzt sich Project Loon auf weitere etablierte Infrastrukturen oder verbindet diese. ${ }^{73}$ Die schwebenden Infrastrukturen sind mit Funkmasten, Glasfaserkabeln und Internetknotenpunkten auf dem Boden verbunden. Google arbeitet mit Netzbetreibern, Kommunikationsdienstleistern und Flughäfen vor Ort zusammen. Trotz dieser verschiedenen materiellen und diskursiven Anschlusspunkte und Überlagerungen kann Google zu einem gewissen Grad losgelöst von diesen operieren, indem der Konzern über eigene mobile Infrastrukturen die Dienstleistungen, an denen er verdient, auch in jenen Regionen zur Verfügung stellt, in denen es keine Kooperationspartner_innen gibt. Das Unternehmen agiert in der Stratosphäre jenseits staatlicher Gesetzgebungen. Damit stellt sich die Stratosphäre von Project Loon als ein extraterritorialer infrastructure space dar, der nicht nur der Überbrückung beschädigter oder nicht vorhandener Infrastrukturen dient, sondern auch in Regionen mit nicht-kooperativen Infrastrukturen zum Einsatz kommt.

Ruhleder und Star haben in ihrem grundlegenden Aufsatz der Infrastructure Studies dafür plädiert zu fragen: «[W]ben - not what - is an infrastructure»? ${ }^{74}$ Diese Umstellung von einer Was- auf eine Wann-Frage, so Gabriele Schabacher, markiere jenen Punkt, «an dem Infrastrukturen zum unsichtbaren Hintergrund alltäglicher Verrichtungen werden». ${ }^{75}$ Transparenz stellen ver-

73 Vgl. Star, Ruhleder: Steps Toward an Ecology of Infrastructure, 112.

74 EBd. 113.

75 Gabriele Schabacher: «Worm World». Infrastruktur, Ökologie und double binds bei Susan Leigh Star und Karen Ruhleder, in: Nadine Taha, Sebastian Gießmann (Hg.): Grenzobjekte und Medienforschung, Bielefeld 2017, 403-418, hier 405.

76 Vgl. Philippe Despoix: Die Welt vermessen. Dispositive der Entdeckungsreise im Zeitalter der Aufklärung, Göttingen 2009, 11.

77 «Balloons Designed to Extend Connectivity", loon.com (17.5.2020).

$78 \mathrm{Vgl}$. loon.com/technology (17.5.2020). meintlich jene Geschichten her, mit denen Googles Marketing-Abteilung jongliert, gelegentlich unterbrochen von Meldungen über Abstürze. Privatisierung und Kommerzialisierung der hohen Himmelsregionen sind auf loon.com in ein Branding verpackt, das an die Tradition aufklärerischer EntdeckungsreiseRhetoriken anschließt und die Stratosphäre als ein neues Jenseits der Kartierung ausweist: ${ }^{76}$ Loons steigen nicht einfach auf, sondern bilden ein «network of balloons traveling on the edge of space», ${ }^{\pi}$ illustriert mit Fotografien von Ballons über kaum besiedelten Regionen (Abb. 8). Unter dem Stichwort «Technology» wird mit Fotografien, Zeichnungen und Videoclips von gut gelaunten Teams und animierten Partyballons das, was das Unternehmen sagt, von dem getrennt, was es macht. ${ }^{78}$ Ballon-Netzwerke als Medieninfrastrukturen, die im Hintergrund funktionieren, zu untersuchen, heißt auch zu beschreiben, in welchen Relationen und Kontexten diese nicht sichtbar geografische Verteilungen, Zugänge 
und Inhalte verzerren. ${ }^{79}$ Project Loon prägt ähnlich den von Starosielski ausgearbeiteten cable geographies spezifische balloon geographies oder bigh-altitude platform geographies aus. ${ }^{80}$ Diese bestehen weniger, wie es Google verspricht, im Ausbau von Reichweiten für Mobilfunkanbieter oder in verbesserter NetzwerkResilienz bei Ausfällen oder Naturkatastrophen. ${ }^{81}$ Vielmehr offenbaren sie die kolonialisierenden Seiten der aufklärerischen Raumexpansions-Rhetoriken und weisen «communities on the periphery of current networks» aus, die «more vulnerable to disconnection or monitoring» bleiben. ${ }^{82}$

Googles Ballon-Netzwerke, wie es der Kulturgeograf Derek McCormack vorgeschlagen hat, als «elemental infrastructure» oder «atmospheric media» zu betrachten, ${ }^{83}$ scheint vor dem Hintergrund der in diesem Aufsatz herausgearbeiteten komplexen materiellen und diskursiven Verwobenheiten stärker an metaphorischen als an rechtlichen, technischen oder wissenschaftlichen Konzepten von Atmosphären orientiert zu sein. Die Installation eines Telekommunikationsnetzes in der Stratosphäre und das Zur-Verfügung-Stellen von Internetdiensten aus der Luft gestalten sich deutlich vielschichtiger, als die Verwendung von Adjektiven wie <elementar> für die Nutzung des Winds als Antrieb in einem staatenlosen Gebiet oder <atmosphärisch> für ubiquitäre und pervasive Informationszirkulationen ohne adressierbares Zentrum es einlösen können. Wie Starosielski dargelegt hat, schöpfen Dienste wie CloudComputing zwar aus der «aerial imagination» drahtloser Datenübertragung, die Wucherung drahtloser Medientechnologien gründet allerdings in einer großen Masse an Kabel. ${ }^{84}$ Die Verwendung von atmosphärischen Metaphern in den Kultur- und Medienwissenschaften legt die Frage nahe, ob diese ähnlichen influential fictions wie das Marketing von Project Loon folgen.
79 Starosielski: Fixed Flow, 65. 80 Starosielski spricht von "cable geographies", vgl. ebd. $81 \mathrm{Vgl}$. loon.com/solutions (17.5.2020).

82 Starosielski: Fixed Flow, 65. 83 Derek P. McCormack: Elemental Infrastructures for Atmospheric Media. On stratospheric variations, value and the commons, in: Environment and Planning, Bd. 35 , Nr. 3, 2017, 418-437. McCormack schließt hier explizit an Marc Hansens Konzept der «atmospheric media" an.

84 Starosielski: Fixed Flow, 53. 